\title{
O PROCESSO DE TRABALHO EM HANSENÍASE: TECNOLOGIAS E ATUAÇÃO DA EQUIPE DE SAÚDE DA FAMÍLIA ${ }^{1}$
}

\author{
Fernanda Moura Lanza², Francisco Carlos Félix Lana ${ }^{3}$
}

\begin{abstract}
${ }^{1}$ Artigo elaborado a partir da dissertação - Tecnologia do processo de trabalho em Hanseníase: análise das ações de controle na microrregião de Almenara, Minas Gerais, apresentada à Escola de Enfermagem da Universidade Federal de Minas Gerais (UFMG), 2009. Pesquisa financiada pelo CNPq - Processo $n^{\circ}$ 40.0785/2005-6.

${ }^{2}$ Doutoranda do Programa de Pós-Graduação em Enfermagem da Escola de Enfermagem da UFMG. Professora Assistente do Curso de Enfermagem da Universidade Federal de São João del Rei, Minas Gerais, Brasil. E-mail: fernandalanza@ufsj.edu.br

${ }^{3}$ Doutor em Enfermagem. Professor Associado do Departamento de Enfermagem Materno-Infantil e Saúde Pública da Escola de Enfermagem da UFMG, Minas Gerais, Brasil. E-mail: xicolana@ufmg.br
\end{abstract}

RESUMO: O estudo teve como objetivo analisar a organização tecnológica do processo de trabalho em hanseníase, empreendido pelos profissionais de saúde que atuam nos serviços da microrregião de Almenara, Minas Gerais. Pesquisa qualitativa, realizada em nove municípios. Como técnicas para a coleta de dados foram utilizadas a entrevista semiestruturada e a pesquisa documental. Foram realizadas 45 entrevistas com gestores e profissionais de saúde. A coleta ocorreu entre novembro 2007 e fevereiro 2008. Para tratamento e análise dos dados foi utilizado a Análise de Conteúdo. Verificamos que os médicos ainda mantêm uma assistência curativa e individual; os enfermeiros dominam todo o processo de trabalho em hanseníase; e os agentes comunitários de saúde realizam as ações educativas, a busca de suspeitos dermatológicos, de contatos e de faltosos. Concluímos que a atual organização dos serviços mostra que ainda existe uma fragilidade nas práticas de saúde voltadas para a abordagem coletiva do problema.

DESCRITORES: Hanseníase. Prevenção \& controle. Atenção primária à saúde. Enfermagem.

\section{THE WORK PROCESS IN LEPROSY: TECHNOLOGY AND THE FAMILY HEALTH TEAM PRACTICE}

\begin{abstract}
The objective of this study is to analyze the technological organization of the work process of leprosy as done by the health workers in the Brazilian Public Health System service units in the municipalities of the Almenara micro-region, in the state of Minas Gerais. This qualitative research was carried out in nine municipalities utilizing semi-structured interviews and document research to collect the data. Forty-five interviews with care providers and health managers were conducted between November, 2007, and February, 2008. Content Analysis was utilized to analyze and treat the data, with results that indicate that physicians still maintain curative and individual care; that nurses dominate the whole process of working in leprosy; and that community health agents carry out educational activities, searching for dermatological symptoms, contacts, and defaulters. We conclude that the current organization of services shows that there is still weakness in the work process in leprosy in order to accomplish the collective approach to the problem. DESCRIPTORS: Leprosy.Prevention \& control. Primary health care. Nursing.
\end{abstract}

\section{EL PROCESO DE TRABAJO PARA TRATAR LA LEPRA: TECNOLOGÍA Y PRÁCTICA DEL EQUIPO DE SALUD FAMILIAR}

RESUMEN: Este estudio tiene el objetivo de analizar la organización tecnológica del proceso de trabajo para tratar la lepra, que es realizado por profesionales que laboran en los servicios de salud de la microrregión de Almenara, en el estado de Minas Gerais, Brasil. Se llevó a cabo una investigación cualitativa en nueve municipios. Se emplearon como técnicas para hacer la recolección de datos la entrevista semi-estructurada y la investigación documental. Se realizaron 45 entrevistas con gestores y profesionales de la salud. La recolección de los datos se hizo entre noviembre de 2007 y febrero de 2008. Para el tratamiento y análisis de los datos se utilizó el Análisis de Contenido. Los resultados indican que los médicos aún emplean una atención curativa e individual, los enfermeros dominan todo el proceso de trabajo para tratar la lepra, y los agentes de salud de la comunidad realizan actividades educativas, búsqueda de síntomas de la piel, de contactos y morosos. Se concluyó que la organización actual de los servicios muestra que todavía hay debilidad en el proceso de trabajo para realizar un enfoque colectivo al problema..

DESCRIPTORES: Lepra. Prevención \& control.Atención primaria de salud. Enfermería. 


\section{INTRODUÇÃO}

O atual cenário da atenção primária à saúde no Brasil está ancorado na Estratégia de Saúde da Família (ESF), que possui um papel fundamental na reorientação do modelo assistencial e na consolidação das diretrizes do Sistema Único de Saúde (SUS). A ESF está pautada em princípios "da família como foco de abordagem, território definido, adscrição da clientela, trabalho em equipe multidisciplinar, co-responsabilização, integralidade, resolutividade, intersetorialidade e estímulo à participação social" 1:19

A publicação da Norma Operacional da Assistência à Saúde (NOAS/SUS 01/2001) foi essencial para a garantia da universalidade e da descentralização da assistência à saúde, regulamentou e ampliou as responsabilidades dos municípios para o acesso da população com equidade a todos os serviços de saúde em todos os níveis de atenção e, na atenção primária, definiu a eliminação da hanseníase como uma das áreas estratégicas de atuação dos serviços de saúde. ${ }^{2}$

A hanseníase ainda se configura como um problema de saúde pública no Brasil, aparecendo nas estatísticas da Organização Mundial de Saúde (OMS) como segundo país em número absoluto de casos detectados em 2009 (37.610). ${ }^{4}$ Em Minas Gerais, a microrregião de Almenara abrange municípios considerados prioritários para o controle da endemia. Dados de 2008 revelam que essa microrregião apresentou uma taxa de prevalência de 4,78 casos de hanseníase/10.000 habitantes e uma detecção de 49,4/100.000 habitantes. Dos 90 casos novos, 53,3\% foram diagnosticados com formas clínicas multibacilares e 2,2\% com grau II de incapacidade física. ${ }^{5}$

As ações de prevenção e controle da doença estão baseadas na realização da detecção oportuna de novos casos, no tratamento com o esquema poliquimioterápico, na vigilância dos contatos domiciliares, na prevenção de incapacidades e na reabilitação. ${ }^{3}$ Para alcançar esses objetivos, é necessário assegurar que as atividades de controle estejam descentralizadas na atenção primária à saúde, representada, no Brasil, pela Estratégia de Saúde da Família.

Na ESF, cada equipe é formada, no mínimo, por um médico generalista, um enfermeiro, um auxiliar de enfermagem e de quatro a seis Agentes Comunitários de Saúde (ACSa), no entanto, as categorias dos profissionais de saúde envolvidos no desenvolvimento das ações de controle da han- seníase podem variar dependendo da estrutura e dos recursos dos serviços de atenção à saúde.

A produção das práticas em saúde, independente do nível de atenção à saúde e das categorias profissionais envolvidas, comporta distintos processos de trabalho, uma vez que a produção de suas práticas é historicamente determinada e fruto das relações estabelecidas entre os diferentes agentes sociais. ${ }^{6}$ No processo de trabalho em saúde, é necessária a utilização de instrumentos não-materiais e materiais para apreensão do objeto de trabalho - que no caso da atenção primária é determinado pelo perfil epidemiológico de uma população evidenciado no território -com a finalidade de produzir a assistência à saúde. ${ }^{6}$

Dessa forma, no processo de trabalho em hanseníase, têm-se como objeto de trabalho os indivíduos ou grupos que podem estar doentes, sadios ou expostos a riscos; os meios de trabalho correspondem aos saberes; e instrumentos utilizados pelos profissionais para alcançar a finalidade deste trabalho, que é realização das ações de prevenção e controle da doença preconizadas pelo Ministério da Saúde. O trabalho em hanseníase deve ser compreendido "como um processo dinâmico e contraditório, que articula a outras atividades do setor saúde e da sociedade e se transforma no atendimento das necessidades sociais".7:27 $\mathrm{O}$ estudo do processo de trabalho, dentro das suas dimensões técnicas e sociais, constitui-se a temática da tecnologia em saúde. ${ }^{8}$

Na microrregião de Almenara, devido a sua endemicidade, a hanseníase configura-se como um objeto do trabalho em saúde. O objetivo desse artigo foi analisar a organização tecnológica do processo de trabalho em hanseníase, empreendido pelos profissionais de saúde que atuam nos serviços da microrregião de Almenara, Minas Gerais.

\section{METODOLOGIA}

Esta pesquisa possui abordagem qualitativa e foi fundamentada no conceito de "Organização Tecnológica do Trabalho". ${ }^{8}$ Essa opção teórico-metodológica foi escolhida por entender ser capaz não só de captar a realidade dos diferentes cenários e sujeitos envolvidos na atenção à hanseníase, trazendo algumas singularidades dessa prática como momentos de uma totalidade mais ampla, mas também, possibilitar a análise da essência da organização tecnológica do trabalho e de seu contexto historicamente determinado. 
A pesquisa foi realizada em nove municípios da microrregião de Almenara, situada no Vale do Jequitinhonha, Estado de Minas Gerais, que possuem diferenças na magnitude da endemia hansênica e da força de transmissão da doença. Os municípios que participaram da pesquisa foram: Almenara, Jacinto, Jequitinhonha, Jordânia, Monte Formoso, Palmópolis, Rubim, Santa Maria do Salto e Salto da Divisa.

Os dados foram coletados em 10 unidades da atenção primária à saúde, sendo todas habilitadas na Estratégia de Saúde da Família e um Centro de Especialidades Médicas, que é referência para a atenção à hanseníase, localizado no município de Almenara.

Como técnicas para a coleta de dados utilizamos a entrevista semiestruturada e a pesquisa documental em registros institucionais e estatísticos. Para obtermos representatividade dos serviços e dos sujeitos, tendo em vista o aprofundamento do objeto em estudo, foram realizadas, no total, 45 entrevistas. Foram convidados os sujeitos que possuem maior representatividade nas práticas de saúde em hanseníase no grupo social em estudo, sendo que em cada município foram entrevistados, no mínimo, um médico, um enfermeiro e um ACS, além dos gestores da saúde. O roteiro da entrevista semiestruturada foi construído de acordo com o sujeito da pesquisa e é composto por duas partes: a primeira parte foi destinada para a identificação do entrevistado e a segunda, composta por questões norteadoras que se referem ao processo de trabalho em hanseníase, os instrumentos utilizados, as ações e finalidades desse trabalho, bem como os fatores facilitadores e dificultadores. Os sujeitos do estudo foram constituídos por 13 gestores, 10 médicos, 12 enfermeiros e 10 ACSs.

A pesquisa documental foi realizada em registros institucionais e estatísticos com o objetivo de corroborar e valorizar as evidências oriundas das entrevistas, colaborando para a apreensão da realidade empírica em suas diversas dimensões. $\mathrm{Na}$ pesquisa documental foram analisadas 111 Atas do Conselho Municipal de Saúde; 10 Planos Municipais de Saúde e 11 Relatórios Finais das Conferências Municipais de Saúde. A coleta de dados ocorreu de novembro 2007 a fevereiro 2008.

Para tratamento e análise dos dados, foi utilizado a Análise de Conteúdo, na modalidade temática. ${ }^{9}$ Foram utilizadas as seguintes identificações dos entrevistados, seguida de um número em ordem crescente, de acordo com o quantitativo de entrevistados por categoria: Gestores $\left(G_{1}\right.$ até $\left.G_{13}\right)$, Médicos $\left(\operatorname{MED}_{1}\right.$ até $\left.M E D_{10}\right)$, Enfermeiros $\left(\mathrm{ENF}_{1}\right.$ até $\left.\mathrm{ENF}_{12}\right)$ e Agentes Comunitários de Saúde $\left(\mathrm{ACS}_{1}\right.$ até $\left.A C \mathrm{~S}_{10}\right)$.

Este estudo foi realizado atendendo às determinações da Resolução 196/96, do Conselho Nacional de Saúde, que estabelece diretrizes e normas regulamentadoras de pesquisas envolvendo seres humanos e foi aprovado no Comitê de ética em Pesquisa (COEP) da Universidade Federal de Minas Gerais, conforme parecer $n^{\circ}$ ETIC 459/05 - Ad 01/07. Esta pesquisa foi financiada com recursos provenientes do Conselho Nacional de Desenvolvimento Científico e Tecnológico (CNPq), através do Edital MCT-CNPq/MS-SCTIE-DECIT-N.35/2005.

\section{RESULTADOS E DISCUSSÃO}

A literatura sugere que a capacidade de diagnosticar os casos de hanseníase está diretamente relacionada ao acesso aos serviços de saúde e ao aumento da oferta das ações integradas à rede básica de saúde. ${ }^{10-11} \mathrm{Na}$ microrregião de Almenara, o processo de descentralização das ações de controle da hanseníase $(\mathrm{ACH})$ para a ESF iniciou-se somente em 2005 e só então foi considerada uma estratégia capaz de enfrentar a endemia hansênica na região. ${ }^{12}$

Nesta pesquisa, sete dos nove municípios possuem uma cobertura total da sua população, pela ESF, inclusive daquelas localizadas na zona rural. Apenas os municípios de Jequitinhonha e Almenara apresentam coberturas populacionais pela ESF inferiores a $100,0 \%$, sendo que, nesse último município, o restante da população não cadastrada é atendida no Centro de Especialidades Médicas. Os resultados apontam que, para o município ter um programa de controle da hanseníase estruturado, não é necessário ter somente a totalidade da população coberta pela ESF, pois esse dado não é sinônimo de cobertura populacional das ações de controle da doença. [...] Jequitinhonha tem vários PSFs, parece que ele tem sete PSFs e quatro ou cinco médicos, enfermeiros, capacitados em hanseníase, certo? Quer dizer, ele tem pessoal treinado para fazer, mas é um pessoal que treinou e num faz diagnóstico, sabe? [...] $\left(\mathrm{G}_{13}\right)$.

Verificamos que o acesso às ações de hanseníase na atenção primária é determinado pela priorização deste agravo na política municipal de saúde, pelo modelo em que é organizada essa assistência, se é descentralizado, centralizado ou um modelo misto, pela presença de profissio- 
nais de saúde capacitados e comprometidos na realização das ações de controle da doença, pela disponibilização de instrumentos para a realização do exame dermato-neurológico, pelo oferecimento do exame baciloscópico e pela disponibilização de recursos para a divulgação dos sinais e sintomas da hanseníase para a população. [...] Nós temos aqui duas unidades hoje que são juntas, uma faz divisa com a outra, estão no mesmo prédio, mas uma faz divisa com a outra em termo de território, uma faz muito diagnóstico e a outra não faz quase nada, entendeu? Então a gente vê assim, às vezes tem um médico que está mais interessado, uma equipe trabalha melhor e a outra não dá muita importância à questão da hanseníase. Às vezes é uma manchinha, mas acaba passando sem ter aquela consideração devida pelos profissionais [...] $\left(\mathrm{G}_{11}\right)$. [...] a nossa divulgação também facilita porque a pessoa sente, quando a pessoa está informada, ela já traz pra gente o problema, né? Isso facilita também [...] $\left(\mathrm{ENF}_{2}\right)$.

Sabe-se que a hanseníase é uma doença endêmica na microrregião de Almenara, constituindo-se assim, como agravo prioritário no planejamento das ações de saúde. Por isso, os gestores, juntamente com os profissionais de saúde e a própria comunidade devem se responsabilizar pelo controle da hanseníase como um problema de saúde pública, ${ }^{11,13-14}$ mas encontramos nos municípios desse estudo uma desigualdade no desenvolvimento das $\mathrm{ACH}$. [...] a gente sabe que a distribuição da hanseníase não é homogênea, mas o que eu coloco pros municípios, que eles é que tem que provar isso, tá certo? Então no momento em que ele tem equipes da Atenção Básica todas treinadas pra fazer diagnóstico, tratamento, prevenção de incapacidades, que ele faça divulgação de sinais e sintomas, porque além dele poder fazer diagnóstico e estar curando o paciente, o paciente têm que reconhecer os sinais e sintomas pra ele buscar o diagnóstico. Então, na verdade, enquanto ele não tiver com isso bem organizado, aí eu costumo dizer pros municípios que o dia quando eles tiverem todas as equipes treinadas, fazendo campanhas de divulgação de sinais e sintomas urbana e rural, utilizando escolas, todos os parceiros, escolas, associações, creches, à medida que eles fizerem essa divulgação ampla por três, quatro anos e tendo as equipes treinadas e que fazem diagnósticos e tendo esse agravo como agravo de relevância local, aí sim, aí é que nós vamos saber quem é quem realmente, se realmente aquele município é um município de poucos casos, ou se realmente aqueles casos, ou seja, existia ali uma prevalência oculta por desconhecimento da população [...] $\left(\mathrm{G}_{13}\right)$.

Verificamos que os municípios de Almenara, Jacinto, Santa Maria do Salto, Rubim, Palmópolis e Jordânia possuem uma concepção mais ampla, tendo o controle da doença como finalidade do trabalho e, para alcançar este fim, realizam as atividades preconizadas pelo Ministério da Saúde para a prevenção e controle da hanseníase. [...] Jaguarão, que é uma comunidade muito próxima de Santo Antônio de Jacinto, [...] a gente está fazendo um trabalho muito intenso e a gente tem um caso de hanseníase, eu estou cabreiro com isso, a gente fez o Dia da Mancha, a gente vai nas fazendas, a gente manda os agentes pras casas [...] Jaguarão a gente está fazendo uma busca ativa assim incansável de mandar os agentes casa por casa, nós fazemos visita domiciliares, a gente dá palestra, a gente vai à igreja [...], só falta colocar um out-door no lugar e os casos não aparecem como em outras regiões [...] $\left(\mathrm{MED}_{10}\right)$.

A sustentação das ações de sensibilização da comunidade é fundamental para alcançar o controle efetivo da doença em função do longo período de incubação da hanseníase, ${ }^{15}$ já que a realização das ações educativas tem como objetivo aumentar a autosuspeição, a detecção de casos e a divulgação dos serviços de saúde. ${ }^{16-17}$ A mobilização de parceiros contribui para a circulação dos conhecimentos sobre a hanseníase em diferentes segmentos sociais e para a prática intersetorial, que é preconizada pelo SUS. ${ }^{16}$

Salto da Divisa e Jequitinhonha realizam basicamente as atividades de diagnóstico e tratamento e deixam em segundo plano, as ações de prevenção. Dessa forma, esses municípios possuem uma concepção fragmentada da finalidade do processo de trabalho em hanseníase. O próprio discurso dos gestores e profissionais confirma que esses municípios estão despreparados para lidar com a hanseníase. [...] não tem trabalho estruturado não, nós não temos, é o que eu te falei, aquié a demanda espontânea. Chegou o caso, a suspeita, a gente providencia, tenta esclarecer o diagnóstico como nossos casos aí todos surgiram de demanda espontânea, quer dizer, nós não temos aquela procura do problema. E o programa de hanseníase reza principalmente essa busca, essa procura desses pacientes que não são descobertos, né? E nós não temos esse programa aqui não. E te confesso, não há um acompanhamento, não há um serviço assim integrado de hanseníase $[. .].\left(\mathrm{MED}_{3}\right)$.

Os profissionais que atuam em Jequitinhonha e Monte Formoso apontam que não existe organização do serviço de saúde e nem capacitação dos profissionais para realizar as ações de controle da hanseníase. Nessas situações, torna-se necessário questionar sobre a real situação epidemiológica da doença ${ }_{1}^{18}$ principalmente em Monte Formoso, 
município que nunca diagnosticou um caso da doença em sua comunidade.

Nos municípios que apresentam dificuldades no desenvolvimento das ACHs, os profissionais de saúde também relataram que a sobrecarga dos outros programas da atenção básica influencia no desenvolvimento das ações do programa de hanseníase. [...] se você me coloca três problemas: puericultura, que era um problema sério com a desnutrição; pré-natal, que também estava sendo um problema sério, pois as mulheres não vinham; e hanseníase. O quê que você acha que eu privilegiei em tentar resolver? Aquilo que eu tinha um pouco mais de chance de dar certo! Peguei a hanseníase, botei debaixo do tapete, vou tentar resolver puericultura e pré-natal. Você tá entendendo a minha situação? Se um, dois, três, quatro, cinco problemas pra resolver, dois deles eu tenho uma chance e três deles é muito mais difícil pra eu conseguir resolver, eu vou fazer de conta que os três não existem e vou privilegiar aqueles dois que eu tenho alguma chance de estar resolvendo [...] $\left(\mathrm{MED}_{9}\right)$.

A sobrecarga de trabalho dos profissionais das unidades da atenção primária do município de Betim, Minas Gerais, também foi identificada como um fator que dificulta o desempenho das ações de controle e o adequado acompanhamento do cliente com hanseníase, mas "a disponibilidade para trabalhar ou não com hanseníase seria inerente ao profissional, parte de um perfil" ${ }^{14: 67}$ Os discursos evidenciaram que há uma grande desigualdade no perfil dos profissionais, sendo que alguns municípios possuem equipes da ESF bem comprometidas com a hanseníase. [...] facilita aqui eu acho que é a questão, primeiro da questão profissional, acho que os profissionais aqui abraçaram a causa mesmo, sabe? Gostam do que tão fazendo, então correm atrás pra que o trabalho seja bem desenvolvido [...] $\left(\mathrm{G}_{9}\right)$.

Em relação ao processo de trabalho na realização das ações de controle da doença, verificamos que existe uma divisão desse processo de trabalho nos serviços de saúde da microrregião. Os médicos ainda mantêm uma assistência curativa e individual ao enfatizarem o seu trabalho para o diagnóstico e a terapêutica, sendo que a maioria deles relatou que não participam de atividades de sensibilização da comunidade e treinamento dos ACSs. [...] O nosso médico ele não está interessado em participar, em fazer palestras com os pacientes e com os agentes de saúde não [...] ( $\left.\mathrm{ACS}_{7}\right)$. [...] quando surge um caso assim suspeito, a enfermeira pega e faz um teste de estesiometria, ai pedimos a bacteriologia pra hanseníase, e depois dos resultados a gente analisa qual tipo de hanseníase e manda a medicação, certo? [...]
$\left(\mathrm{MED}_{5}\right)$. A literatura descreve que é muito comum o baixo envolvimento dos profissionais médicos no desempenho das ações de controle da hanseníase. ${ }^{15}$

Verificamos que a avaliação dermatoneurológica é, muitas vezes, realizada pelo médico juntamente com a enfermeira, devido a pouca experiência dos profissionais no manejo da hanseníase. [...] marco um momento eu e a enfermeira juntas. Primeira coisa que eu faço, entendeu? Por vários motivos, primeiro, nem eu enem a enfermeira antes do curso tinha muita vivência com hanseníase e duas mentes pensam melhor que uma, então eu e a enfermeira juntas, a gente teria mais condições de diagnosticar do que eu sozinha ou ela sozinha [...], e como nem eu e nem a enfermeira temos grandes experiências com o exame físico do paciente hanseniano, eu procuro, eu tento fazer com que o paciente passe por nós duas juntas [...] $\left(\mathrm{M}_{\mathrm{q}}\right)$. Este relato evidencia que os atuais esforços para realizar o treinamento dos profissionais da ESF para desenvolver as ações de prevenção e controle da hanseníase nem sempre resultam nas equipes confiança suficiente para diagnosticar a doença ou iniciar o esquema terapêutico. ${ }^{11}$ Por outro lado, a interação entre os membros da ESF promove modos alternativos de atuar na realização das ações preconizadas pelos protocolos de hanseníase do Ministério da Saúde.

É interessante destacarmos a experiência de Rubim e Jacinto que realizam reuniões para discussões de casos, com o intuito de manter a equipe sempre mobilizada em relação à doença e também para resolver as dúvidas dos profissionais na condução dos casos. [...] depois que foi feito todos os treinamentos, que a gente pegou um pouco mais de manuseio da questão da hanseníase, a gente reuniu e achou que era uma boa idéia pra gente estar discutindo o caso de não ficar aquela questão de ficar, é eu aprender sozinho, sem muita experiência, ficar na dúvida, o outro colega atender sozinho, sem muita experiência, ficar na dúvida. A gente montou um grupo que às sextas-feiras à tarde a gente traz os casos de cada área e faz um atendimento em conjunto. Dentro da sala vai estar presente às vezes dois, às vezes três médicos, uma enfermeira, duas enfermeiras fazendo o exame completo do paciente [...] $\left(\mathrm{MED}_{4}\right)$.

O diagnóstico é confirmado em uma consulta realizada por um grupo de profissionais, não por falta de capacidade técnica dos profissionais, mas como forma de aumentar a experiência no manejo da hanseníase e também para reforçar o aprendizado teórico. A discussão de casos com profissionais mais experientes é uma oportunidade para obter melhor qualidade do trabalho em saúde, além de ser uma atividade de treinamento de pessoal. ${ }^{8}$ 
O município de Jacinto também capacitou os odontólogos para realizarem a suspeita diagnóstica de hanseníase, durante o exame clínico de rotina, a fim de que todos os profissionais contribuam para o controle da endemia no município. Os odontólogos podem suspeitar de lesões de pele em áreas visíveis como braços e pernas, além das lesões características de casos multibacilares como madarose e infiltrações na testa, nariz e orelhas, e das lesões hansênicas que podem estar presentes no palato de indivíduos com hanseníase.

O profissional enfermeiro tem um papel essencial no processo de trabalho em hanseníase, já que é o responsável pelo planejamento e execução das ações de assistência e controle dos pacientes e dos contatos. É também responsável por realizar o primeiro atendimento a esse cliente hanseniano. Assim, ele tem um papel importantíssimo na escuta, na valorização das queixas e na educação em saúde: [...] eu recebo o paciente e explico pra ele, primeira coisa que eu faço, o primeiro instrumento que eu acho é a palavra, que a gente usa pra está explicando pro paciente sobre tudo sobre a doença, inclusive forma de tratamento e o que ela pode causar no organismo, as consequências de não tratar, tudo isso. Pra depois partir pro exame físico [...] $\left(\mathrm{ENF}_{10}\right)$.

As tecnologias não-materiais são utilizadas pelos enfermeiros para valorizar a fala e a escuta durante os atendimentos, produzir vínculos e responsabilizade para com os usuários. Esses são alguns elementos capazes de reorientar o processo de trabalho em saúde. ${ }^{20}$

O envolvimento do enfermeiro na realização das ações de controle da hanseníase ampliou-se consideravelmente após a implantação da poliquimioterapia, já que iniciaram a execução de novas tarefas como a supervisão da dose mensal e avaliação de incapacidades físicas. ${ }^{21}$ "A enfermeira realiza as orientações ao usuário sobre como devem ser tomadas as medicações, buscando mantê-lo informado sobre como seguir seu tratamento, sendo esta uma maneira de promover a adesão ao mesmo, em longo prazo. Mais do que retornar ao posto para tomar as medicações sob a supervisão de um profissional, é enfatizado que a sua presença regular no serviço de saúde é importante para um acompanhamento contínuo da história de seu problema de saúde, de como ele está reagindo ao tratamento, das queixas, das alterações e das demais necessidades que ele possa apresentar" . 17:294

As doses supervisionadas são administradas a cada 28 dias pelo enfermeiro e, excepcionalmente, pelos auxiliares de enfermagem e ACSs. No ato do comparecimento à unidade de saúde para receber a medicação específica preconizada, a dose supervisionada, o paciente deve ser submetido a uma consulta para a identificação precoce de estados reacionais, efeitos colaterais ou adversos aos medicamentos em uso, desenvolvimento de incapacidades físicas, surgimento de dano neural, além de ser uma oportunidade para promover a educação em saúde. ${ }^{11}[. .$.$] a gente pega o prontuário,$ né? Faz a anamnese na pessoa, vê como que ela está, né? Pergunta se está tomando o remédio direitinho e anota as queixas, pesa, né? Sinais vitais [...] $\left(\mathrm{ENF}_{4}\right)$.

O enfermeiro também é responsável pela supervisão do trabalho dos ACSs. Verificamos assim que o processo de trabalho dos profissionais da ESF é fragmentado. Mesmo tendo o profissional médico como membro da equipe, o profissional enfermeiro detém sozinho a supervisão da equipe de auxiliares de enfermagem e ACSs, realização das ações de vigilância epidemiológica e programação local. ${ }^{8,22}$

Nos municípios mais engajados com a hanseníase, os ACSs participam ativamente das atividades de controle da hanseníase: fazem suspeita diagnóstica durante a visita domiciliar, encaminham o paciente em caso de reações, realizam orientações sobre prevenção de incapacidades, buscam os contatos e os faltosos, e, em situações especiais, supervisionam o tratamento. [...] eu tive uma pessoa que eu descobri porque ele simplesmente me disse que ele podia tomar qualquer coisa quente, colocar na mão que ele não sentiria a dor. Aí eu virei pra ele e falei: o senhor poderia me emprestar a sua mão e o senhor olhar pro lado? Ele falou: posso. Aí eu peguei a mão dele, ele olhou pro lado, eu peguei o bico do meu lápis que estava com a ponta bem fina e apertei profundamente e ele não sentiu nada [...] $\left(\mathrm{ACS}_{\mathrm{q}}\right)$. Para desenvolver essas atividades, os ACSs utilizam os conhecimentos técnicos referentes aos sinais e sintomas da hanseníase, que são as "tecnologias não-materiais". 8

O trabalho dos agentes comunitários, durante as visitas domiciliares, é de suma importância na efetivação das $\mathrm{ACHs}$, devido a sua proximidade com a população, ${ }^{19}$ e esse profissional pode ser eleito como o principal executor da busca ativa de suspeitos dermatológicos na população. A visita domiciliar é um instrumento de trabalho do ACS, que permite a construção de vínculos de confiança com as famílias, a observação de seus hábitos, a detecção de problemas, a troca de informações e a transmissão de orientações em saúde. ${ }^{23}$

Dessa forma, as orientações realizadas pelos ACSs, durante as visitas domiciliares, podem ser consideradas como a principal estratégia para pro- 
mover e manter a sensibilização da comunidade em relação à hanseníase, já que essas atividades possuem uma periodicidade sistemática e alcançam a totalidade da população adscrita em cada microárea.

Ao analisar a forma como os profissionais de saúde envolvidos no controle da hanseníase se organizam para a produção de suas práticas, verificamos que a atual organização dos serviços de saúde dos municípios está estruturada pelo modelo clínico que visa o atendimento individual do doente, sendo que as práticas de saúde voltadas para a abordagem coletiva do problema (educação para a comunidade, busca dos contatos, vigilância epidemiológica) ainda são bastante pontuais. Apesar dessa fragilidade, as práticas de abordagem coletiva da hanseníase se articulam com as práticas individuais para dar sentido à totalidade do processo de trabalho em hanseníase.

Em outro cenário também foi evidenciada a predominância do discurso anátomo-clínico na prática de enfermagem em hanseníase, limitando-se o atendimento ao corpo biológico ${ }^{17} \mathrm{~A}$ implantação do processo de enfermagem pode fortalecer o atendimento integral ao doente de hanseníase já que, na etapa de coleta de dados, poderão ser abordados aspectos afetivos, sociais, econômicos e hábitos de vida, sem, no entanto, deixar de evidenciar os aspectos específicos da hanseníase como o exame dermato-neurológico e a avaliação de incapacidades. ${ }^{24}$ No entanto, ainda existe uma "carência de profissionais aptos a trabalhar com a concepção de saúde ampliada" . ${ }^{20: 234}$

O que dificulta a continuidade das ações de controle da hanseníase em todos os municípios deste estudo, que possuem essas ações implantadas, é a rotatividade dos profissionais. [...] um problema que eu acho é rotatividade de profissional. Agora mesmo, nós estamos com três profissionais que chegaram recentemente, que ainda não foi capacitado pra questão de hanseníase, e também isso dificulta o trabalho, então a gente tem que parar um pouquinho pra capacitar e eles comecem a andar junto com os que já estão desenvolvendo o trabalho [...] $\left(\mathrm{G}_{9}\right)$. [...] Em 2006 nós tivemos uma queda [do número de casos novos], porque nós tivemos um período de turbulência, saiu vários profissionais da rede, enfermeiros e médicos, entraram muitos novos, então isso deu uma queda, deu uma esfriada ao processo de descentralização $[\ldots]\left(\mathrm{G}_{11}\right)$.

A rotatividade dos profissionais é o grande inimigo das ações de controle da hanseníase e, inclusive, reflete negativamente na condição de saúde da população. ${ }^{25-26}$ Por isso, a Gerência Regional de Saúde de Pedra Azul tem que estar planejando atualizações constantes das equipes da microrregião, para manter acesa a" chama da hanseníase", que ainda está muito longe de alcançar o controle da endemia, pois as dificuldades operacionais do programa de controle da doença estão muito relacionadas à ausência de profissionais treinados, ${ }^{23} \mathrm{e}$ comprometidos com a hanseníase.

Alguns municípios da microrregião empregam estratégias específicas para enfrentar os obstáculos frente ao processo de controle da hanseníase como problema de saúde pública, tais como a realização de cursos de capacitação para os profissionais recém-contratados, tendo como monitores os próprios profissionais que atuam nas equipes da ESF, e as reuniões clínicas, que possibilitam a construção do conhecimento das ações de controle da hanseníase na própria prática de seus agentes.

As reuniões clínicas, uma tecnologia desenvolvida no processo de trabalho em hanseníase, nos municípios desse estudo, se constituem em uma estratégia de educação continuada porque, durante as discussões, há aquisição de conhecimento técnico, já que os profissionais mais experientes no manejo da doença ajudam a solucionar as dúvidas dos demais integrantes. ${ }^{8}$ Assim, as equipes de supervisão são uma alternativa para os municípios sustentarem o treinamento nas ações de prevenção e controle da hanseníase. ${ }^{11,14,27}$ Essas iniciativas revelam a preocupação dos gestores para manter a capacitação dos profissionais de saúde no que diz respeito às ações de diagnóstico e tratamento de hanseníase. ${ }^{19}$

Mas é evidente que os cursos de capacitação não conseguem acompanhar a rotatividade dos profissionais. ${ }^{13}$ Por outro lado, esse profissional capacitado que saiu de um município, vai ser contratado por outro. O novo município o recebe sensibilizado para a hanseníase, e se esse profissional tem responsabilidade social com os agravos que acometem uma população, independente de qual for o município, espera-se que ele dará uma resposta a essa necessidade, implantando ou implementando as ações de prevenção e controle da hanseníase.

A manutenção de um número apropriado de profissionais de saúde capacitados e qualificados para desempenhar as ações de controle da hanseníase é hoje a luta dos municípios de regiões endêmicas e consiste no principal pilar em prol do controle epidemiológico da doença e da sustentação das ações do programa na atenção primária à saúde. ${ }^{27}$ 


\section{CONSIDERAÇÕES FINAIS}

Verificamos o empenho dos municípios de Almenara, Jacinto, Santa Maria do Salto, Rubim, Palmópolis e Jordânia em realizar as ações de controle da hanseníase de acordo com as normas técnicas e protocolos do Ministério da Saúde. Esperava-se que, com a integração das ações de controle na atenção primária, o modelo de atenção à hanseníase tivesse incorporado os conceitos de vigilância à saúde.

As práticas dos profissionais da Estratégia de Saúde da Família na atenção à hanseníase ainda são orientadas pelo modelo clínico: os médicos realizam basicamente o diagnóstico e tratamento da hanseníase, os enfermeiros, a avaliação dermatoneurológica, a avaliação e a prevenção de incapacidades, além daadministração da dose supervisionada e outros, os agentes comunitários de saúde desempenham práticas de orientações diversas ao doente, e os odontólogos que, no município de Jacinto, foram capacitados para realizarem a suspeita dermatológica.

Vale ressaltar a importância do trabalho em equipe para a construção do conhecimento na abordagem ao paciente de hanseníase. Observamos, em alguns municípios, que é possível realizar essa abordagem de forma compartilhada entre médicos, enfermeiros e ACSs.

A realização de práticas de controle da doença no território extra-muro da unidade de saúde, como o domić́lio, reforça os conceitos de uma nova forma de "fazer saúde". No entanto, a abordagem dos aspectos sociais, econômicos e hábitos de vida dos doentes precisam ser sistematicamente valorizados nos atendimentos dos profissionais da atenção primária.

A partir dos resultados encontrados, concluímos que a estrutura e organização dos serviços de saúde têm grande influência no desenho da situação epidemiológica da doença nessa microrregião do Vale do Jequitinhonha. As atividades de controle da hanseníase, que são ofertadas pelos serviços de saúde dos municípios da microrregião de Almenara, são influenciadas pelo nível de organização tecnológica desses serviços e que as práticas de saúde em hanseníase são tecnologias desenvolvidas dentro do próprio processo de trabalho e são historicamente e socialmente determinadas, pois ocorrem de formas distintas de acordo com o local e com as transformações que ocorrem na sociedade.

Esperamos que os resultados dessa pesquisa possam despertar uma reflexão crítica dos gestores e profissionais de saúde para reorganizarem o processo de trabalho em hanseníase na microrregião. O principal desafio dos municípios desse estudo é a reestruturação do programa de controle da doença fundamentada em políticas públicas efetivas que garantam a sustentabilidade econômica, política e social do plano de controle da hanseníase.

\section{REFERÊNCIAS}

1. Conselho Nacional de Secretários de Saúde (BR). Atenção primária e promoção da saúde. Brasília (DF): CONASS; 2007.

2. Brasil. Portaria n. 95 de 26 de janeiro de 2001. Norma Operacional da Assistência à Saúde NOAS/SUS 01/2001. Brasília (DF): Ministério da Saúde; 2001.

3. Brasil. Portaria n. 3.125 de 7 de outubro de 2010: aprova as Diretrizes para Vigilância, Atenção e Controle da hanseníase [online]. [acesso 2011 Abr 3]. Disponível em: http://portal.saude.gov.br/ portal/arquivos/pdf/formularios_portaria_n3125 hanseniase.pdf

4. World Health Organization. Global leprosy situation 2010. Wkly Epidemiol Rec. 2010 Aug; 85(35): 337-48.

5. Secretaria de Estado da Saúde (MG). Coordenadoria de Controle da Hanseníase. Seminário Estadual 2009. Belo Horizonte (MG): SES; 2009.

6. Mishima SM, Villa TCS, Silva EM, Gomes ELR, Anselmi ML, Pinto IC, et al. Organização do processo gerencial no trabalho em saúde pública. In: Almeida $\mathrm{MCP}$, Rocha SMM, organizadores. O trabalho de enfermagem. São Paulo (SP): Cortez; 1997. p. 251-296.

7. Villa TCS, Mishima SM, Rocha SMM. A enfermagem nos serviços de saúde pública do Estado de São Paulo. In: Almeida MCP, Rocha SMM, organizadores. O trabalho de enfermagem. São Paulo (SP): Cortez; 1997. p. 27-60.

8. Mendes-Gonçalves RBM. Tecnologia e organização social das práticas de saúde: características tecnológicas do processo de trabalho na rede estadual de centros de saúde de São Paulo. São Paulo (SP): HUCITEC; 1994.

9. Bardin L. Análise de conteúdo. Lisboa (PT): Edições 70; 1977.

10. Penna MLF, Oliveira MLW, Carmo EH, Penna GO, Temporão JG. Influência do aumento do acesso à atenção básica no comportamento da taxa de detecção de hanseníase de 1980 a 2006. Rev Soc Bras Med Trop. 2008; 41(Supl II): 6-10.

11. Visschedijk J, Engelhard A, Lever P, Grossi MAF, Feenstra P. Leprosy control strategies and the integration of health services: an international perspective. Cad Saúde Pública. 2003 Nov-Dez; 19(6):1567-81. 
12. Lanza FM, Lana FCF. Decentralization of leprosy control actions in the micro-region of almenara, state of Minas Gerais. Rev Latino-am Enfermagem. 2011 Jan-Fev; 19(1):187-94.

13. Brandão P. Assistência ao portador de hanseníase. Rev Bras Enferm. 2008; 61(Esp):782-3.

14. Fuzikawa PL. Avaliação da descentralização das ações de controle da hanseníase em Betim: prevenindo incapacidades? [dissertação]. Belo Horizonte (MG): Universidade Federal de Minas Gerais, Faculdade de Medicina; 2007.

15. Silva AR, Portela EGL, Matos WB, Silva CCB, Gonçalves EGR. Hanseníase no município de Buriticupu, Estado do Maranhão: busca ativa na população estudantil. Rev Soc Bras Med Trop. 2007 Nov-Dez; 4 (6):657-60.

16. Kelly-Santos A, Monteiro SS, Ribeiro APG. Collection of educational materials on Hansen's disease: a tool for memory and communicative practices. Interface. 2010 Jan-Mar; 14(32):37-51.

17. Silva FRF, Costa ALRC, Araújo LFS, Bellato R. Prática de enfermagem na condição crônica decorrente de hanseníase. Texto Contexto Enferm. 2009 Abr-Jun; 18(2):233-40.

18. Helene LMF, Pedrazzani ES, Martins CL, Vieira CSCA, Pereira AJ. Organização de serviços de saúde na eliminação da hanseníase em municípios do Estado de São Paulo. Rev Bras Enferm. 2008; 61 (spe): 744-752.

19. Moreira TMA. Avaliação da descentralização das ações programáticas de hanseníase: um estudo de caso [tese]. Rio de Janeiro (RJ): Escola Nacional de Saúde Pública, Fundação Oswaldo Cruz; 2002.
20. Pessanha RV, Cunha FTS. A aprendizagem-trabalho e as tecnologias de saúde na Estratégia Saúde da Família. Texto Contexto Enferm. 2009 Abr-Jun; 18(2):233-40.

21. Oliveira MLW. O papel estratégico do enfermeiro no controle da hanseníase. Rev Bras Enferm. 2008; 61(spe):668.

22. Nascimento MS, Nascimento MAA. Prática da enfermeira no Programa de Saúde da Família: a interface da vigilância da saúde versus as ações programáticas em saúde. Ciênc Saúde Coletiva. 2005 Abr-Jun; 10(2):333-45.

23. Lara MO. Configuração identitária do agente comunitário de saúde de áreas rurais [dissertação]. Belo Horizonte (MG): Escola de Enfermagem, Universidade Federal de Minas Gerais; 2008.

24. Duarte MTC, Ayres JA, Simonetti JP. Consulta de enfermagem: estratégia de cuidado ao portador de hanseníase em atenção primária. Texto Contexto Enferm. 2009 Jan-Mar; 18(1):100-7.

25. Kalk A, Fleischer K. The decentralization of the health system in Colombia and Brazil and its impact on leprosy control. Lepr Rev. 2004 Mar; 7(1):67-78.

26. Borrelli FRG. A rotatividade dos profissionais de saúde na zona rural de Sergipe: um problema a ser enfrentado [dissertação]. Rio de Janeiro (RJ): Escola Nacional de Saúde Pública, Fundação Oswaldo Cruz; 2004.

27. Saunderson P. Learning to manage leprosy after 2005: preserving critical knowledge and exploiting new technology. Lepr Rev 2005 Mar; 76(1):2-4. 